\title{
Photoinduced ultrafast local volume changes in intermediate-valence solids
}

\author{
Momar S. Diakhate and Martin E. Garcia \\ Theoretische Physik, FB 18 and Center for Interdisciplinary Nanostructure Science and Technology (CINSaT), Universität Kassel, \\ Heinrich-Plett-Str. 40, 34132 Kassel, Germany
}

(Received 4 August 2008; revised manuscript received 26 February 2009; published 27 March 2009)

\begin{abstract}
We present a theoretical model for the description of the ultrafast structural response of intermediate-valence solids to femtosecond laser excitation. The approach includes the calculation of the free energy of the hot electrons produced by the laser pulse and the determination of the changes in the thermodynamic and elastic properties of the solid as a consequence of the excitation. In particular and based on the promotional RamirezFalicov model, we consider the femtosecond laser heating of $\alpha$ cerium and the subsequent ultrafast lattice expansion dynamics. For this purpose, we determine the thermodynamic properties of cerium at very high electronic temperatures (simulating the laser excitation). The possibility for a nonequilibrium photoinduced inverse volume collapse transition is discussed. We consider both the laser-excited and the unexcited parts of the system in order to account for inertial confinement. The thermodynamic properties are obtained as function of time and used to calculate the shock velocity variation and the time scale for expansion of the heated spot into the surrounding (unheated) part of the sample. A transition on a subpicosecond time scale is predicted.
\end{abstract}

DOI: 10.1103/PhysRevB.79.094117

PACS number(s): 33.20.Xx, 05.70.-a, 71.28.+d

\section{INTRODUCTION}

Intense femtosecond laser pulses are able to produce in solids a situation of extreme nonequilibrium in which electrons acquire very high temperatures while the lattice remains cold. The laser-excited electrons modify the potential landscape which governs the motion of the ions, causing lattice instabilities and structural transitions ${ }^{1,2}$ In the last decade a literal explosion of investigations on laser-induced structural phase transitions has taken place. Different theoretical and experimental studies of laser-induced ultrafast solid-liquid $^{3-5}$ and solid-solid ${ }^{6-12}$ phase transitions have been recently reported.

A common feature of most of the observed laser-induced ultrafast structural changes is that the irradiated region in the material exerts pressure on the surrounding unexcited part. This can be easily understood by taking into account that the laser excites electrons into antibonding states in the case of covalent solids or simply produces an increase in the electronic pressure of metallic systems. However, the expansion velocity of the irradiated part is limited by the sound velocity. Consequently, volume changes are expected to occur on much longer time scales than those on which the laserinduced structural transformation takes place. A particularly interesting case may arise when both time scales become comparable. Therefore, for systems undergoing isostructural volume collapse transitions, such as cerium, one would expect an interesting dynamics of volume changes upon femtosecond laser excitation. The physical behavior of cerium and mixed-valence rare-earth systems based on $\mathrm{Ce}, \mathrm{Sm}, \mathrm{Eu}$, and $\mathrm{Yb}$ has been the subject of many theoretical and experimental works during the last few decades.

This interest is due to the fact that such materials show an isostructural first-order transition under certain conditions of pressure and temperature, ending in a critical point. At room temperature this transition is accompanied by a dramatic and discontinuous volume change of $\sim 14 \%$ at $p \sim 8 \mathrm{kbar}$. Because of the large volume change all the interpretations of the existing experimental data agree on the fact that the transition requires a qualitative change in the $4 f$ electrons.

Several theories have been proposed in order to describe the electronic and magnetic properties of rare-earth metals under different external conditions. All of them are valuable in giving information on particular aspects of the problem but differ on the description of the electronic structure behavior near the phase boundary. Despite the controversy concerning the mechanism of the volume collapse transition, all these theories give evidence that because of its isostructural nature (both phases exhibit a fcc structure), the transition is purely electronically driven. Promotional type theories explain the transition as being produced by electron delocalization due to a transition from the $4 f$ level into the $5 d 6 \mathrm{~s}$ conduction band. Hence, the existence of the isostructural transition depends crucially on the orbital degeneracy of the $f$ states.

In this paper we present a theoretical study of the structural response of $\mathrm{Ce}$ upon femtosecond laser excitation and predict a very fast laser-induced inverse volume collapse transition from the $\alpha$ to the $\gamma$ phase. We compute the free energy and expansion velocity of laser-excited Ce based on a simple microscopic model which qualitatively accounts for the changes in the electronic structure due to the action of the laser pulse. The model, developed by Ramirez and Falicov, ${ }^{13}$ only considers electron localization and delocalization in a Mott-type picture and does not include recent modern improvements, which are briefly described at the beginning of Sec. II. Nevertheless, our model gives a qualitative description of the effect of the isostructural volume change on the electronic system.

We simulate the laser excitation by a sudden increase in the electronic temperature. Thus, we obtain the equation of state (EOS) in the heated part of the material from the Helmholtz free energy of the system and calculate the $P-V-T$ diagram. The change in temperature due to the laser is accompanied by change in pressure which causes the expansion of the heated part through the development of a shock front which produces changes in the unexcited part of the sample 


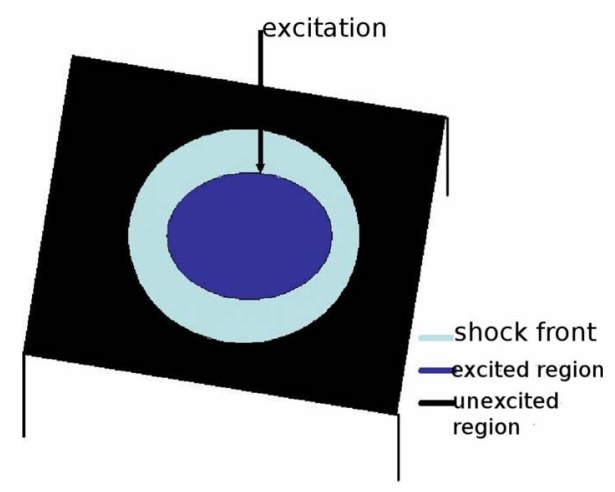

FIG. 1. (Color online). Scheme of the mechanical change induced in $\alpha$-Ce by a femtosecond laser pulse. The unexcited part of the system remains cold and is compressed with the shock velocity driven by the pressure $P$ of the excited region, which is high due to the excitation. In the calculations, the pressure is assumed to be continuous at the interface between expanding and shocked matters.

(see Fig. 1). Assuming the total volume to be constant, the change in volume of the expanded part must be compensated by a compression of the surroundings. The abrupt change in the pressure of the shock-wave front (i.e., the boundary between the shocked region and the nonshocked region) due to the increase in temperature causes dramatic variations in the velocity, stress, and density of the material. The excited region exerts mechanical pressure in the shocked front which causes material compression in the unexcited region. We simulate such material deformation processes using the Hugoniot theory. ${ }^{14}$

The paper is organized as follows. In Sec. II we present the theoretical model on which this work is based. Results are presented and discussed in Sec. III. We predict a fast inverse volume collapse transition for Ce. Finally, in Sec. IV we draw conclusions on the time scales and summarize the most important points.

\section{THEORY}

The calculations were done in the framework of the model proposed by Ramirez and Falicov, ${ }^{13}$ which is based on the existence of two possible and qualitatively different states for the electrons: the localized $f$ electronic states (ioniclike states) and the itinerant $d$-band electronic states, coupled via an effective intra-atomic Coulomb repulsion $G$. The volume collapse transition is then described by a delocalization of the electrons through a promotion of electrons from the $f$ to the $4 d$ states.

It is important to point out that more recent theories are able to account for more degrees of freedom than the promotional model and are able to describe the alpha-gamma transition as an entropy driven process. ${ }^{15,16}$ Both the nonmagnetic ( $\alpha$ phase) and magnetic ( $\gamma$ phase) phases are interpreted, respectively, as pure Kondo phases with high and low Kondo temperatures $T_{K} \cdot{ }^{17,18}$ According to the modern descriptions not the occupancy of the $f$ electrons but its spectral weight shifts as the temperature is raised due to the unlocking of the Kondo state. ${ }^{19}$ The most important fact is, however, that the volume collapse transition is accompanied by electron delocalization, as one would intuitively expect. The delocalization manifests itself by an increase in double occupancies of the $d$ orbitals. ${ }^{20}$

The above-mentioned improvements certainly contributed to obtain a more accurate picture of the electronic properties and, in particular, of the excitation spectrum of $\mathrm{Ce}$ at low temperatures. However, and concerning the equation of state $p(V T)$, the promotional model still yields correct results. Therefore, it also reproduces the values of the sound velocity and mechanical properties of $\mathrm{Ce}$ with reasonable accuracy. Since our calculations are based on these quantities, we expect our results to give a correct qualitative description of the laser-induced phenomena in $\mathrm{Ce}$.

Note also that the Kondo effect has been shown to be important for a quantitative description of the transition in thermodynamical equilibrium. However, in the case of laserinduced transitions the electronic temperature $\left(T_{\mathrm{el}}\right)$ generated by the excitation is far above the Kondo temperature of $\mathrm{Ce}$ $\left(T_{K}\right)$. Therefore, effects involving much lower energy scales compared to the absorbed energy can be neglected.

The electronic Hamiltonian is given by the sum of three different terms,

$$
H=\sum_{k} \epsilon_{k} d_{k}^{+} d_{k}+G \sum_{i} f_{i}^{+} f_{i} d_{i}^{+} d_{i}+\epsilon_{f} \sum_{i} f_{i}^{+} f_{i},
$$

with $d_{k}^{+}$and $d_{k}$ being the creation and annihilation operators for $s-d$ electrons in the conduction-band states with energy $\epsilon_{k} . f_{i}^{+}$and $f i$ are the creation and annihilation operators for a $f$ state electron at the lattice site $i$, with binding energy $\boldsymbol{\epsilon}_{f}$. A double occupancy of the localized $f$ state is excluded. The first term in the Hamiltonian is the contribution to the energy coming from the itinerant conduction electrons. The second term represents the on-site Coulomb interaction between localized $f$ states with number density $n_{f}=(1 / N) \sum_{i} f_{i}^{+} f_{i}$ and the $s-d$ band electrons with number density $n_{c}=(1 / N) \sum_{i} d_{i}^{+} d_{i}$, where $N$ is the total number of atoms. Finally, the third term refers to the energy of localized $f$ electrons (ionic energy).

If the system is in thermodynamical equilibrium it is straightforward to obtain the equation of state from the Helmholtz free energy, including the contributions from the electrons and the ions. For the calculation of thermodynamic properties upon femtosecond laser excitation we propose the following model presented below.

\section{A. Model for laser heating of Ce}

As mentioned in Sec. I, a femtosecond laser induces in the material an extreme nonequilibrium state in which the electron and lattice temperatures differ dramatically. The formation of this state can be qualitatively understood as follows. First, electrons gain a large amount of energy due to the absorption of photons. As a consequence, a nonequilibrium (non-Fermi-type) electron distribution is produced which, due to electron-electron collisions, thermalizes after a short time to a Fermi-type electron distribution $f\left(T_{\mathrm{el}}\right)$, with $T_{\mathrm{el}}$ being the electron temperature after laser excitation. Note that $T_{\mathrm{el}}$ is much higher than the room temperature, reflecting the laser heating of electrons. Consequently, $T_{\mathrm{el}} \gg T_{\text {ionic }} \simeq T_{\text {room }}$ 
since both laser excitation and electron thermalization occur on time scales which are shorter typical phonon periods. ${ }^{21}$ Therefore, while the electrons acquire a temperature of a few thousand kelvin, the ions remain at room temperature $T_{\text {room }}$. The large increase in $T_{\mathrm{el}}$ leads to an increase in the electron pressure. As a consequence, the laser-excited region tends to expand against the surrounding unexcited part. The resulting expansion dynamics, which determines the laser-induced inverse volume collapse transition, is microscopically described in the following.

Using the mean-field approximation, the electronic part of the Helmholtz free energy per atom for a given volume $V$ and electronic temperature $T_{\mathrm{el}}$ is given by

$$
F(T, V)=F_{\text {conf }}(T, V)+F_{\text {el }}(T, V)+F_{\text {mag }}(T, V) .
$$

Here, the first term $F_{\text {conf }}$ refers to the electronic contribution to the ionic configuration, the second one $F_{\mathrm{el}}$ accounts for the free energy of the conduction electrons, whereas the last term describes the magnetic free energy. The density of states is assumed to be uniform and normalized in such a way as to allow four valence electrons in the ground state. The conduction band extends from $-D / 3$ to $2 D / 3$, with $D=8.16 \mathrm{eV}$ being the bandwidth. The Fermi energy is taken to be zero $\left(E_{F}=0\right)$.

In the ground state, the occupied part of the conduction band has a width of $2.72 \mathrm{eV}$ while the width of the empty part is $5.44 \mathrm{eV}$. The electronic part of the free energy can be expressed as $F_{\mathrm{el}}=E_{\mathrm{el}}-T S_{\mathrm{el}}$, with from $E_{F}$,

$$
E_{\mathrm{el}}=\int n(\epsilon, V) f\left(\epsilon, T_{\mathrm{el}}\right) \epsilon d \epsilon,
$$

and

$$
\begin{aligned}
S_{\mathrm{el}}\left(T_{\mathrm{el}}, V\right)= & \int n(\epsilon, V)\left\{f\left(\epsilon, T_{\mathrm{el}}\right) \ln f\left(\epsilon, T_{\mathrm{el}}\right)\right. \\
& \left.+\left[1-f\left(\epsilon, T_{\mathrm{el}}\right)\right] \ln \left[1-f\left(\epsilon, T_{\mathrm{el}}\right)\right]\right\} d \epsilon .
\end{aligned}
$$

Here, $f\left(\epsilon, T_{\mathrm{el}}\right)$ denotes the Fermi-Dirac distribution and $n(\epsilon, v)$ denotes the electronic density of states. The chemical potential entering in $f\left(\epsilon, T_{\mathrm{el}}\right)$ is computed using the fact that the number of electrons per atom remains constant upon laser excitation, i.e., $n_{f}+n_{c}=4$, with

$$
n_{c}=\frac{1}{N} \int n(\epsilon, V) f\left(\epsilon, T_{\mathrm{el}}\right) d \epsilon
$$

being the total number of conduction electrons and $n_{f}$ as the occupation number of $f$ level. The magnetic free energy, second term of Eq. (2), is written as

$$
F_{\text {mag }}(T, v)=-k_{B} T n_{f} \ln [2 J+1],
$$

where $2 J+1=6$ is the spin degeneracy and $J=5 / 2$ is the total angular momentum of the localized $4 f$ electron.

Finally, the configurational free energy $F_{\text {conf }}=E_{\text {conf }}$ $-T S_{\text {conf }}$ is given by

$$
F_{\text {ions }}=\epsilon_{f} n_{f}-G n_{f}^{2}-k_{B} T\left[n_{f} \ln \left(n_{f}\right)+\left(1-n_{f}\right) \ln \left(1-n_{f}\right)\right] \text {. }
$$

Here, the first two terms represent the mean ionic energy of the ground-state configuration of Ce ions and $n_{f}$ is the occupation number of the localized $4 f$ orbitals. We recall that the $f$ electrons are assumed to be well localized on the ions. The last term refers to the configurational entropy of the localized $f$ electrons, which is given by the different possible distributions of the $n_{f}$ electrons on the ions.

\section{B. Laser-induced volume changes}

In order to describe the lattice expansion dynamics we assume, based on the original idea of Stampfli and Bennemann, ${ }^{1,2}$ that on subpicosecond time scales there is practically no entropy exchange between electrons and ions, and therefore the electronic entropy remains constant;

$$
S_{\mathrm{el}}\left(T_{\mathrm{el}}, V\right)=S_{0} .
$$

This assumption is correct as long as only very few degrees of freedom of the lattice become active after laser excitation. For instance, it has been successfully used to determine the dynamics of coherent phonons in $\mathrm{Bi}^{22}$ In the case of laser-excited $\mathrm{Ce}$ in the $\alpha$ phase it is reasonable to assume that the motion of the lattice immediately after femtosecond excitation is the expansion and that other types of lattice motion only become active on later times. Therefore, we rely on Eq. (8) and use it to determine the electronic temperature $T_{\mathrm{el}}$ as a function of time.

Thus, the excited part of the system will expand through the formation of a shock wave (see below), where the unexcited part will compress. This is shown schematically in Fig. 1. The compression of the unexcited part is not adiabatic since the whole sample is supposed to be kept at the temperature at which the experiment is performed (for instance, room temperature). The electronic pressure which builds up in the excited part as a consequence of the laser excitation is calculated as

$$
P=-\left.\frac{\partial E_{\mathrm{el}}}{\partial V}\right|_{S} .
$$

When the excited part expands the on-site energy of the $f$ orbitals must increase since the attractive potential of the neighboring ions becomes less important. We model this fact by writing $\epsilon_{f}=\epsilon_{f}^{0}+\lambda\left(V-V_{0}\right) . \epsilon_{f}^{0}$ is the $f$-level position in the absence of volume changes and $\lambda$ is an adjustable parameter, which is determined from our choice of $\epsilon_{f}^{\alpha}$ and $\epsilon_{f}^{\gamma}$. The temperature dependence of the thermodynamic quantities is obtained from the free energy $F\left(T_{\mathrm{el}}, v\right)$ for the $f$-level occupation is computed using ${ }^{23} n_{f}=-\frac{\partial F}{\partial \epsilon_{f}}$. The first step of the simulation is to determine the chemical potential from charge conservation $\left(n_{c}+n_{f}\right)=4$. Then, the other thermodynamic quantities $n_{f}, E, p \ldots$ are calculated. As mentioned before, the sudden increase in the electronic temperature of the laserexcited part leads to a rapid increase in the electron pressure. As a consequence, the excited part of the system will expand. Due to the large difference in temperature and entropy 
TABLE I. Fitting parameters using data from Ref. 24 for $u_{p}$ $\neq 0$.

\begin{tabular}{lcc}
\hline \hline$c_{0}$ & $c_{1}$ & $c_{2}$ \\
\hline 0.6052 & 2.4765 & -0.2292 \\
\hline \hline
\end{tabular}

and carrier density between the excited and the nonexcited regions, nonlinear effects are expected to be important and the expansion takes place through the build up of a shock wave. In order to account for this effect we describe the shock wave by using the Rankin-Hugoniot equations, which are derived from the three conservation laws (mass, momentum, and energy, respectively). ${ }^{24}$

$$
\begin{gathered}
\rho\left(u_{s}-u_{p}\right)=\rho_{0} u_{s}, \\
P-P_{0}=\rho_{0} u_{s} u_{p}, \\
E-E_{0}=\frac{1}{2}\left(P+P_{0}\right)\left(V-V_{0}\right) .
\end{gathered}
$$

Here the subscript 0 labels the quantities in the unexcited solid. $E$ refers to the internal energy and $\rho_{0}$ refers to the initial density. Note that the complete process of shock-wave propagation in solids can be governed by the above three conservation laws. The combination of the three conservation laws mentioned above yields the transformation between the kinetic quantities (particle- and shock velocities) and the thermodynamic variables $(P, V, E)$. In the first equation (10), $u_{s}$ and $u_{p}$ refer to the shock and the so-called particle velocity, respectively. They are related by the following equation: ${ }^{24}$

$$
u_{s}=c_{0}+c_{1} u_{p}+c_{2} u_{p}^{2},
$$

where the coefficients $c_{0}, c_{1}$, and $c_{2}$ are determined from the experimental data by a least-squares fitting method, and for the particular case of Ce they are shown in Table I. This method for deriving the isotherm in the cold part of the material is based on the analysis of the Hugoniot curve, where the quantities $u_{s}$ and $u_{p}$ can be directly measured. Combining Eqs. (11) and (10) leads to the relation between the applied pressure and particle velocity across the shock front. It is assumed that compression behind the shock line is isotropic and that the cold part of the material is in thermodynamic equilibrium. Proposed relationship (11) is valid in the range of pressure and temperature considered in the present work. The high pressure induced by the laser pulse in the heated region leads, according to Eq. (10), to an increase in the particle velocity. We assume that the particle velocity across the front is equal to the velocity of the material behind the front. In other words, we assume that the expansion velocity of the heated part is equal to compression velocity of the unexcited part. Thus, we evaluate the lattice-volume change in the vicinity of the shock front from the time integration of the particle velocity,

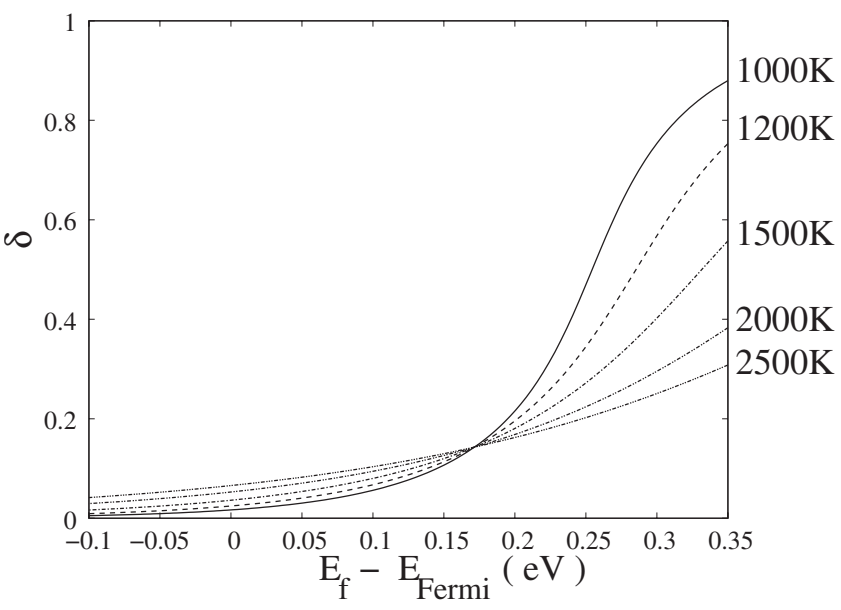

FIG. 2. Degree of delocalization, $\delta$, as function of $f$-level position in the band for different values of electronic temperature $T_{\mathrm{el}}$. The $f$-level position is determined with respect to the Fermi energy. The degree of delocalization $\delta$ depends strongly on the $f$-level occupation in the band in the vicinity of the Fermi level.

$$
\delta l=2 \int_{0}^{t} u_{p} d t
$$

With a constant total volume, and assuming isotropic expansion, this equation serves to determine the volume change in the expanded material. As already stated above, the shock velocity is derived from pressure by combining Eq. (11) and the standard relations of the three laws, conservation equation (10). The results obtained using these calculations are presented in Sec. III.

\section{RESULTS AND DISCUSSION}

We performed numerical calculations on the basis of the model described in Sec. II. In this section we analyze the volume changes and the changes in the degree of electron delocalization, which we define as

$$
\delta=1-n_{f}
$$

In Fig. 2 we show the development of the degree of electron delocalization at different electronic temperature depending on the $f$-level position. As mentioned before, high electronic temperatures correspond to high laser fluences. We have found that the decrease in delocalization of the $4 f$ states increases with increasing $\boldsymbol{\epsilon}_{f}$. The delocalization of the $4 f$ electrons takes place continuously in the high volume gamma phase. This is consistent with recent density functional theory (DFT) calculations. ${ }^{20}$

Since the $f$-level position will change upon the laserinduced expansion, the curve shows that, particularly at high electronic temperatures, no discontinuous transition will occur. This means that for the conditions created by the laser pulse the $5 d 6 s-4 f$ transition seems to occur continuously. For a sufficiently large value of $\epsilon_{f}$, no $f$ occupation is expected.

As explained in Sec. II, the time evolution of the system was introduced using the shock-wave compression technique. The detailed mechanism of the shock compression of 


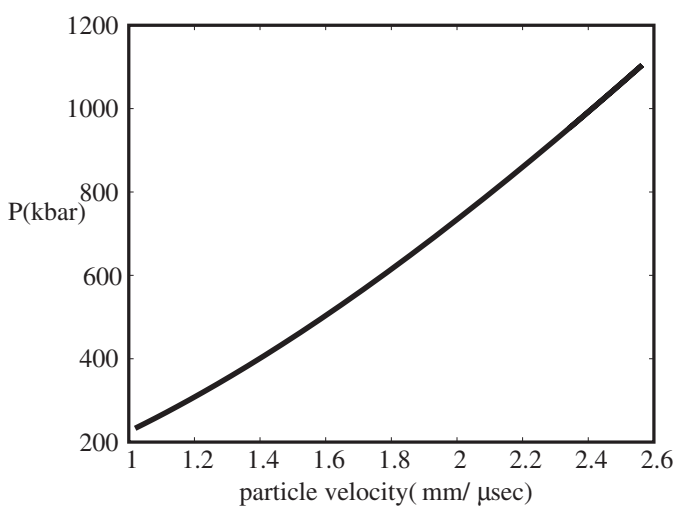

FIG. 3. Plot of pressure vs particle velocity in the cold material obtained in the present work. The particle velocity $u_{p}$ is assumed to be constant in the bulk.

solids has not yet been clarified. However, the shock-wave compression technique remains a very important tool to study solids under shock loading. Since solids are incompressible in practical terms, very large pressures are necessary to create even a modest change in volume.

In general, solids do not behave like fluids at low stress due to the presence of shear stress. This means that solid stress and plastic flow may enhance the mechanical changes. This is what we expect to occur upon shock-wave formation induced by a femtosecond laser pulse.

The increase in the electronic temperature induced by the femtosecond laser pulse leads to the increase in the pressure. This results in the formation of shock waves and in the expansion of the system. To describe the dynamics of the system and in particular depropagation of the shock waves after the laser heating, and for the sake of simplicity, we assume the volume expansion to be isotropic.

We assume that the total volume of the sample remains constant within the time scales on which the shock waves propagate. This assumption is clearly valid since only a small part of the sample is irradiated (the size of the laser spot is usually considerably smaller than surface of the sample). Thus, thermal expansion of the whole sample will be a negligible effect. Moreover, it will also occur on much longer time scales than those involved in the shock-wave formation and propagation.

Since the total volume remains constant, and as explained in Sec. II, the change in volume of the heated part due to expansion is equal to the change in the unexcited part due to compression. Note that the time scale for the expansion of the excited region will be governed by the compression velocity in the unexcited part.

We have calculated the laser-induced pressure from Eq. (9) and used Eqs. (10) and (11) to determine the particle velocity $u_{p}$. The resulting system of four equations with the four unknowns $\left(u_{s}, u_{p}, P\right.$, and $\left.V\right)$ is solved numerically. In Fig. 3 we plot the interdependence between $u_{p}$ and $P$.

It is important to mention that we do not consider the effect of the elastic pressure in our calculations. This is because the elastic term in the free energy [not considered in Eq. (2)] will lead to volume oscillations at time scales which are much larger than those which are relevant for this work.

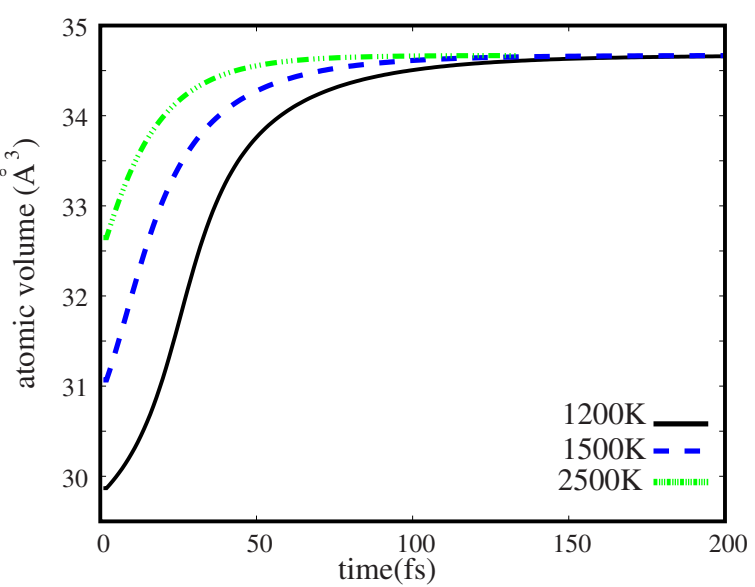

FIG. 4. (Color online) Volume variation as a function of time after laser excitation for different electronic temperatures. At the maximum reached volume the corresponding degree of delocalization is almost 0 . Note that for higher electronic temperatures the volume expansion is faster.

In Fig. 4 the time evolution of the volume of the excited part is shown for different electronic temperatures (corresponding to different laser fluences). We obtain very fast volume changes on the order of $15 \%$ occurring on a time scale of $150 \mathrm{fs}$. This shows that ultrashort laser pulses of high-enough intensity can induce ultrafast local volume changes in Ce. Interestingly, these sudden laser-induced volume changes are related to a transition from $\alpha$-Ce to $\gamma$-Ce. As a consequence of the expansion there is a continuous transition in the occupation of the $4 f$ level from values near $n_{f} \sim 0$ to $n_{f} \rightarrow 1$. Thus, we predict a laser-induced inverse volume collapse transition or a laser-induced localization of the valence electrons on a subpicosecond time scale.

In Fig. 5 we plot the time evolution of the degree of electron delocalization $\delta$ assuming an excitation which leads to an electronic temperature of $T_{\mathrm{el}}=1000 \mathrm{~K}$. The very fast

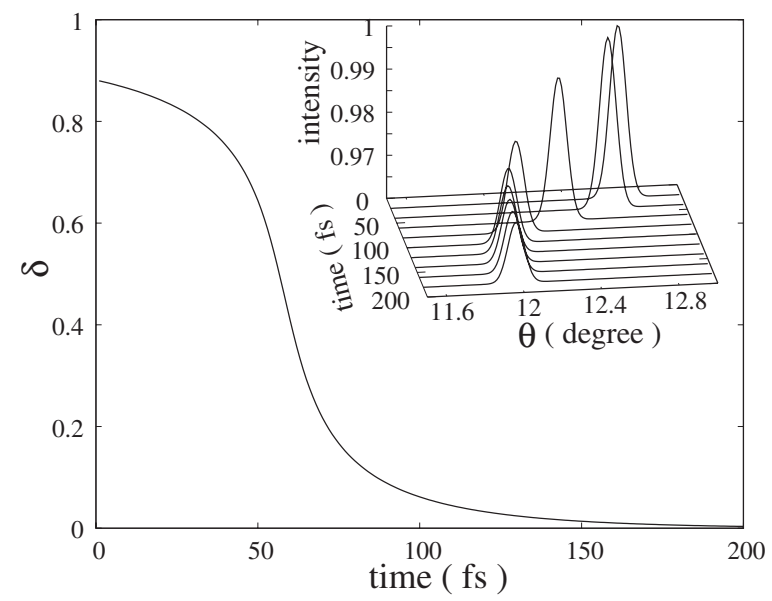

FIG. 5. Time evolution of the electron delocalization degree for a laser excitation resulting in an electronic temperature $T_{\mathrm{el}}$ $=1000 \mathrm{~K}$. The initial time $t=0$ corresponds to the laser excitation. Note that for this high electronic temperature no discontinuity is observed. The inset shows the time evolution of the [111] Bragg peak obtained from Eq. (14). 
localization of the electrons yields to the volume changes shown in Fig. 4.

The structural part of the transition (volume expansion) could be monitored via time-dependent rocking curves measured through pump-probe experiments based on probe ultrashort $\mathrm{x}$-ray pulses $^{7}$ or through time-resolved electron diffraction. ${ }^{10,25}$ It is well established that for fcc crystals the (111) Bragg peak is that of highest intensity,.., Therefore we have computed the time dependence of the Bragg reflections during the lattice expansion from the structure factor,,

$$
|I(t)|^{2}=\left|\frac{1}{N} \sum_{i=1}^{N} e^{i G_{k h l} \cdot R_{i}(t)}\right|^{2},
$$

where $G_{k h l}$ is the corresponding reciprocal lattice vector and $R_{i}(t)$ is the position of ion $i$ in the unit cell at time, $t$., The diffraction angle is obtained from the Bragg condition,.,

In the inset of Fig. 5 is the time evolution of the (111) Bragg peak. Note that there is a very rapid shift of the peak toward smaller angles reflecting the laser-induced expansion.

The time evolution of the pressure $P$ after the laser excitation derived from the free energy applying Eq. (9) is shown in Fig. 6 for different values of the electronic temperature $T_{\mathrm{el}}$. Note, the presence of a maximum for a given time, which corresponds to the change in curvature for the time behavior of the volume (Fig. 4). This maximum shifts to shorter times as the laser fluence increases.

\section{SUMMARY}

We used a microscopic model of laser-induced structural changes combined with an electronic model Hamiltonian and the Hugoniot theory to demonstrate that an ultrafast photoinduced transition involving large local volume and electronic changes can be induced in cerium. In the range of

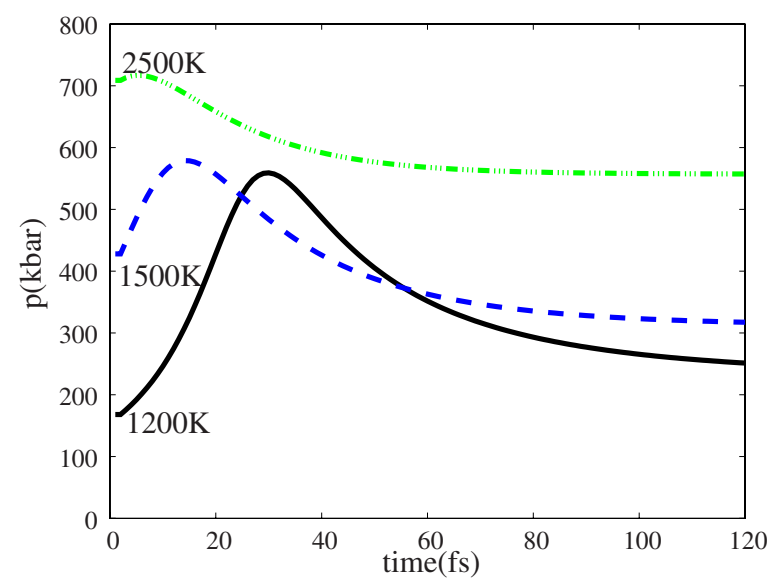

FIG. 6. (Color online) Time evolution of the total pressure for different electronic temperatures.

electronic temperatures we have considered in this work, the photoinduced transition occurs as rapid expansion of the solid, initially in the high density $\alpha$ phase, due to the strong excitation of the band electrons. This expansion is accompanied by electron delocalization. We predict a very fast expansion dynamics helped by the formation of shock waves. As a consequence, an ultrafast shift of the (111)-Bragg peak occurs which should is possible to be measured by means of time-resolved diffraction experiments.

\section{ACKNOWLEDGMENTS}

This work has been supported by the Deutsche Forschungsgemeinschaft (DFG) through priority Program No. SPP 1134 and by the BMBF through the Verbundprojekt No. FSP301-FLASH (FKZ: 05KS7SJ1).
${ }^{1}$ P. Stampfli and K. H. Bennemann, Phys. Rev. B 42, 7163 (1990).

${ }^{2}$ P. Stampfli and K. H. Bennemann, Phys. Rev. B 46, 10686 (1992).

${ }^{3}$ P. L. Silvestrelli, A. Alavi, M. Parrinello, and D. Frenkel, Phys. Rev. Lett. 77, 3149 (1996).

${ }^{4}$ S. L. Johnson, P. A. Heimann, A. M. Lindenberg, H. O. Jeschke, M. E. Garcia, Z. Chang, R. W. Lee, J. J. Rehr, and R. W. Falcone, Phys. Rev. Lett. 91, 157403 (2003).

${ }^{5}$ A. M. Lindenberg et al., Science 308, 392 (2005).

${ }^{6}$ H. O. Jeschke, M. E. Garcia, and K. H. Bennemann, Phys. Rev. B 60, R3701 (1999).

${ }^{7}$ A. Cavalleri, Cs. Toth, C. W. Siders, J. A. Squier, F. Raksi, P. Forget, and J. C. Kieffer, Phys. Rev. Lett. 87, 237401 (2001).

${ }^{8}$ C. Kübler, H. Ehrke, R. Huber, R. Lopez, A. Halabica, R. F. Haglund, and A. Leitenstorfer, Phys. Rev. Lett. 99, 116401 (2007).

${ }^{9}$ H. O. Jeschke, M. E. Garcia, and K. H. Bennemann, Phys. Rev. Lett. 87, 015003 (2001)

${ }^{10}$ F. Carbone, P. Baum, P. Rudolf, and A. H. Zewail, Phys. Rev.
Lett. 100, 035501 (2008).

${ }^{11}$ M. Rini, R. Tobey, N. Dean, J. Itatani, Y. Tomioka, Y. Tokura, R. W. Schoenlein, and A. Cavalleri, Nature (London) 449, 72 (2007).

${ }^{12}$ R. Kitagawa, H. Takebe, and K. Morinaga, Appl. Phys. Lett. 82, 3641 (2003).

${ }^{13}$ R. Ramirez and L. M. Falicov, Phys. Rev. B 3, 2425 (1971).

${ }^{14}$ Barry R. Krueger and Thad Vreeland, Jr., J. Appl. Phys. 69, 710 (1991).

${ }^{15}$ M. Lüders, A. Ernst, M. Däne, Z. Szotek, A. Svane, D. Ködderitzsch, W. Hergert, B. L. Györffy, and W. M. Temmerman, Phys. Rev. B 71, 205109 (2005).

${ }^{16}$ B. Amadon, S. Biermann, A. Georges, and F. Aryasetiawan, Phys. Rev. Lett. 96, 066402 (2006).

${ }^{17}$ J. W. Allen and L. Z. Liu, Phys. Rev. B 46, 5047 (1992).

${ }^{18}$ J. W. Allen and R. M. Martin, Phys. Rev. Lett. 49, 1106 (1982).

${ }^{19}$ K. Haule, V. Oudovenko, S. Y. Savrasov, and G. Kotliar, Phys. Rev. Lett. 94, 036401 (2005).

${ }^{20}$ K. Held, A. K. McMahan, and R. T. Scalettar, Phys. Rev. Lett. 87, 276404 (2001). 
${ }^{21}$ H. O. Jeschke and M. E. Garcia, in Nonlinear Optics, Quantum Optics and Ultrafast Phenomena with X-rays, edited by B. W. Adams (Kluwer Academic, London, 2003).

${ }^{22}$ E. S. Zijlstra, L. L. Tatarinova, and M. E. Garcia, Phys. Rev. B 74, 220301(R) (2006).
${ }^{23}$ Pavol Farkašovski, Phys. Rev. B 54, 7865 (1996).

${ }^{24}$ W. H. Gust and E. B. Royce, Phys. Rev. B 8, 3595 (1973).

${ }^{25}$ M. Harb, R. Ernstorfer, C. T. Hebeisen, G. Sciaini, W. Peng, T. Dartigalongue, M. A. Eriksson, M. G. Lagally, S. G. Kruglik, and R. J. Dwayne Miller, Phys. Rev. Lett. 100, 155504 (2008). 